\title{
Spatiotemporal gait patterns during overt and covert evaluation in patients with Parkinson's disease and healthy subjects: is there a hawthorne effect?
}

\author{
Verónica Robles-García ${ }^{1,2}$, Yoanna Corral-Bergantiños ${ }^{1,2}$, Nelson Espinosa ${ }^{1,2}$, María \\ Amalia Jácome $^{1}$, Carlos García-Sancho ${ }^{3}$, Javier Cudeiro ${ }^{1,2}$, Pablo Arias ${ }^{1,2}$ \\ ${ }^{1}$ Universidade da Coruña $;{ }^{2}$ Institute of Biomedical Research of A Coruña $;{ }^{3}$ Complexo Hospitalario Universitario \\ de A Coruña
}

\begin{abstract}
Parkinson's disease (PD) and aging lead to gait impairments. Some of the disturbances of gait are focused on step length, cadence, and temporal variability of gait cycle. Under experimental conditions gait can be overtly evaluated, but patients with PD are prone to expectancy effects; thus it seems relevant to determine if such evaluation truly reflects the spontaneous gait pattern in such patients, and also in healthy subjects. Thirty subjects (15 subjects with PD and 15 healthy control subjects) were asked to walk using their natural, preferred gait pattern. In half of the trials subjects were made aware that they were being evaluated (overt evaluation), while in the rest of the trials the evaluation was performed covertly (covert evaluation). During covert evaluation the gait pattern was modified in all groups. Gait speed was significantly increased $(P=.022)$; step cadence and average step length were also significantly modified, the average step length increased $(P=.002)$ and the cadence was reduced $(P \leq .001)$. Stride cycle time variability was unchanged significantly $(P=.084)$. These changes were not significantly different compared between elderly and young healthy controls either. Due to the small sample size, a note of caution is in order; however, the significant results suggest that covert evaluation of gait might be considered to complement experimental evaluations of gait.
\end{abstract}

Keywords: Parkinson's disease, elderly, walking, methods, effect modifier 
Gait impairments are a cardinal sign in Parkinson's disease (PD) and are present also in aging. ${ }^{1,2}$ A classic kinematic evaluation of gait focuses on gait speed, cadence, and step length; stride time variability is also of interest, since it is correlated with the risk of falling. ${ }^{1-6}$

Gait evaluation procedures may themselves influence performance, ${ }^{7}$ and the awareness of being evaluated (Hawthorne effect ${ }^{8,9}$ ) and/or being engaged in a gait task might modify execution, becoming unrepresentative of daily living gait. In this respect, Morris et $\mathrm{al}^{10}$ evaluated gait covertly in a group of patients with PD to control for placebo effects after an intervention based on visual cues. They showed a positive treatment effect in overt trials. However, whether it was induced by treatment expectancy or by the Hawthorne effect was not clearly discerned. The healthy group did not show differences between overt and covert gait evaluation in absence of visual cues; in this case, the effect of treatment expectancy was absent.

Hence, it seems relevant to determine the spontaneous execution of gait in the absence of overt evaluation in patients with PD, without the influence of any treatment. Although this point is sometimes considered by clinicians, it has not been addressed during experimental evaluation.

Here we investigate the putative effect of overt evaluation on parkinsonian spatiotemporal gait parameters by comparing participants' walking patterns when they are aware versus unaware of being evaluated (overt and covert evaluation, respectively). Our hypothesis is that overt evaluation causes subjects to modify gait patterns from the spontaneous covert gait.

\section{Methods}

\section{Participants}

We recruited a sample of 30 subjects. Fifteen nondemented patients with idiopathic PD were evaluated OFF-dose; ages ranged from 57 to 87 years (mean 68.20; 11 males) and the mean unified Parkinson's disease rating scale (UPDRS) score was 33.20 points (ranging from 13 to 52 points). Fifteen healthy subjects were also studied. To account for a putative effect of aging on the outcomes, this sample included young (mean $24.87 \mathrm{y}$, ages ranged from 21 to $37 ; \mathrm{n}=8,1$ male) and elderly (mean $64.00 \mathrm{y}$, ages ranged from 55 to $71 ; \mathrm{n}=7,2$ males) subgroups. Procedures were approved by the University of A Coruna ethical committee.

\section{Protocol}

The protocol was bound to the Helsinki declaration, and subjects signed consent forms. Subjects were asked to walk normally on level ground for $22 \mathrm{~m}$ along a predefined track, having been informed that evaluation would be undertaken (overt evaluation) (Figure 1). Only data from the central $17 \mathrm{~m}$ were analyzed. Once they reached the end of the corridor, they were informed that the trial had ended, asked to go back to the start point, and wait for the following trial. However, recording was maintained while subjects went back to the starting position, though they were unaware of being evaluated (covert evaluation). A total of 13 trials in 8 subjects were discarded because their behavior distorted the normal performance of gait (stopping, speaking, and others). A number of 13 trials were added in for these subjects to have 5 of each (overt and covert) for analysis. 


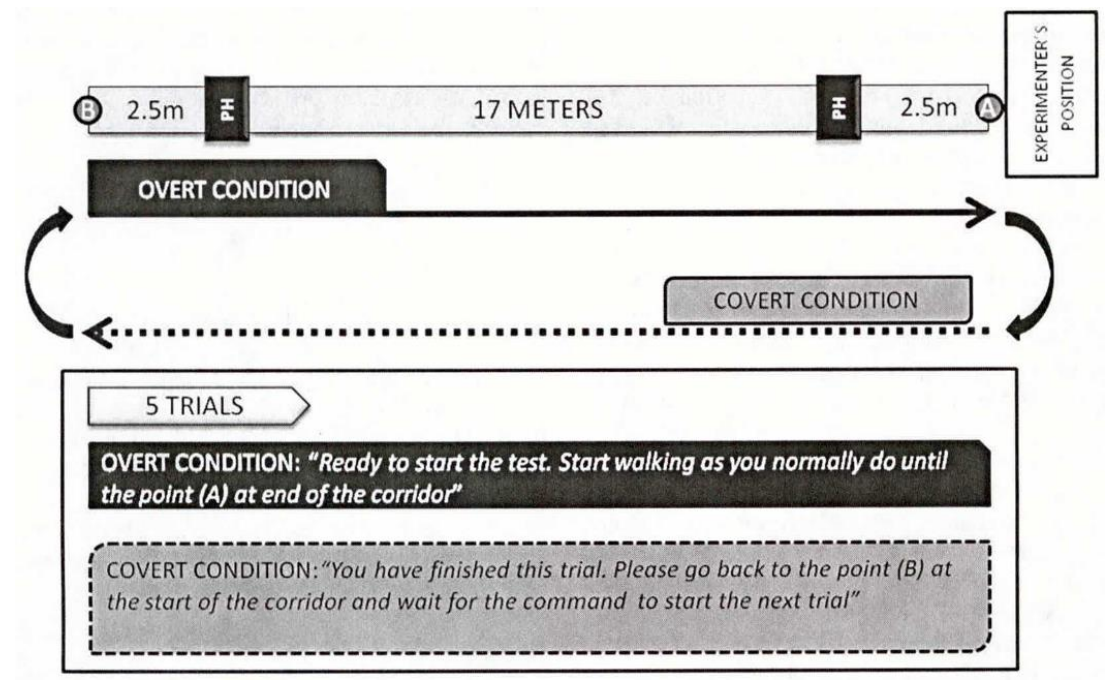

Figure 1 - Experimental protocol. Subjects were asked to walk along a 22-m corridor. Two photocells (PH) were placed $17 \mathrm{~m}$ apart, leaving $2.5 \mathrm{~m}$ on each side to allow turns, and to obtain steady gait pattern recordings. The figure explains the instructions given to the subjects. When the subjects returned to the start point (point B) (covert evaluation) they were not able to see the experimenter

\section{Material}

Gait spatiotemporal parameters were acquired by means of 2 photocells placed $17 \mathrm{~m}$ apart at each side of the corridor and foot switches to detect contact times (sample at $1 \mathrm{KHz}$ ) (Biometrics Ltd., Newport, UK).

\section{Outcome Variables}

Gait speed $(\mathrm{m} / \mathrm{s})$ was calculated from the time taken to cover the $17 \mathrm{~m}$. We obtained cadence (steps/s) and stride time variability (coefficient of variation $[\mathrm{CV}], \mathrm{CV}_{\text {stride-time }}$ ) from contact times. The average step length $(\mathrm{m})$ was calculated from gait speed and cadence. $\mathrm{CV}_{\text {stride-time }}$ was defined as $\mathrm{CV}_{\text {stride-time }}(\%)=$ (SD/mean) x 100, and expresses arrhythmicity in stride temporal pattern. Gait outcomes were calculated from the mean of the 5 trials.

\section{Statistical Analysis}

Differences in the groups' ages were tested with $t$ tests. Each of the 4 kinematic variables was analyzed by means of an ANOVA with repeated measures, with 1 within-subjects factor (overt, covert) and 1 between-subjects factor group with 3 levels (PD, elderly control, young control). We also computed the partial eta square $\left(\eta^{2} p\right)$ to estimate the magnitude of the effects. Observed values were expressed by the mean and $95 \%$ confidence intervals (CI). Due to the reduced sample size in the different groups, the statistical test might be not sensitive enough to detect interaction of overt-covert effect between group factors. Therefore, to check the influence of the sample size on the significance of the interaction effects, we conducted a post hoc power analysis to calculate the smallest mean difference needed for which power exceeded 95\% as follows: we computed the pooled SD of the within-subject covert-overt differences from each SD and the sample sizes, found the minimum effect sizes for which the design had 95\% power for the comparisons between groups in the context of a one-way ANOVA, and converted them into the smallest mean differences required to detect differences among groups in the covert-overt 
differences with $95 \%$ power. For this purpose we used the statistical package G*Power 3.1.9 (University of Düsseldorf, Düsseldorf, Germany). Normality of distributions was assessed by Kolmogorov-SmirnovLilliefors and Shapiro-Wilk tests. Significance was set at $\mathrm{P} \leq .05$.

\section{Results}

There was no statistically significant difference in age between PD subjects and elderly controls $(P=$ $.225)$, but young controls were significantly younger than PD subjects $(P<.001)$. The ages of young and elderly controls were not compared statistically since age was the basis of their grouping.

Some analyzed variables changed significantly from overt to covert; this was the case for gait speed $\left(\mathrm{F}_{1,27}=5.909 ; P=.022 . \eta^{2}=.18\right)$, step cadence $\left(\mathrm{F}_{1,27}=17.013 ; P \leq .001, \eta^{2}{ }_{p}=.387\right)$, and step length $\left(\mathrm{F}_{1,27}=11.426 ; P=.002, \eta_{p}^{2}=.297\right)$. For the $\mathrm{CV}_{\text {stride-time, }}$, the differences between overt and covert evaluation were not significant $\left(\mathrm{F}_{1,27}=3.213 ; P=.084, \eta^{2}{ }_{p}=.106\right)$. Figure 2 displays these variables pooled and split by group, while Figure 3 displays these variables by individual points.

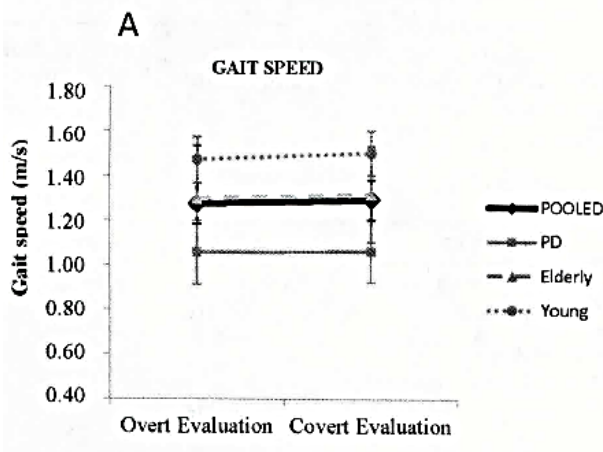

C

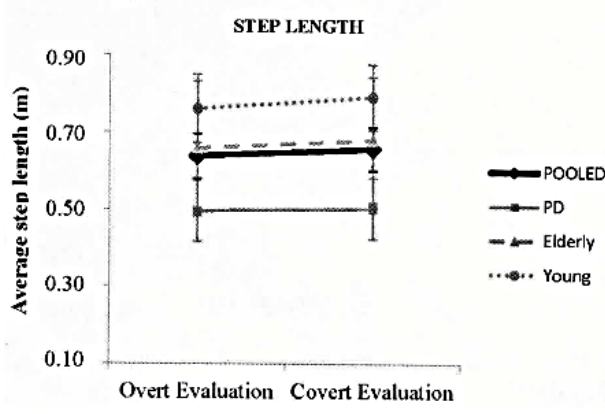

B

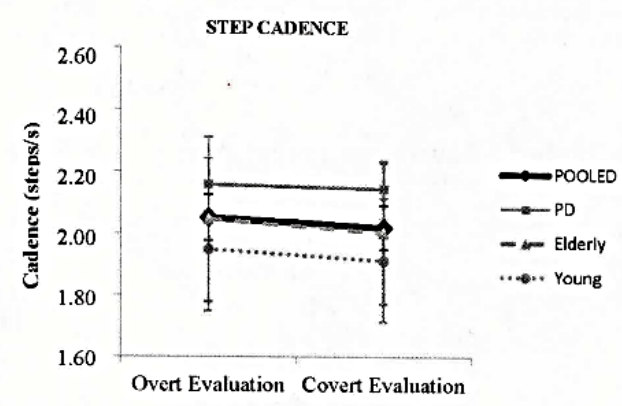

D

cV

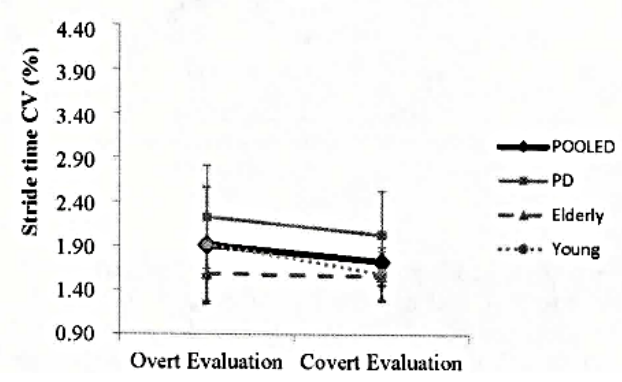

Figure 2 - Effect of overt and covert evaluation on gait speed (A), step cadence (B), step length (C), and $\mathrm{CV}_{\text {stride-time }}$ (D). The figure shows the effect of covert evaluation on the kinematic variables in the 3 groups (light solid lines for PD subjects, dashed lines for elderly controls, and dotted lines for young controls); the thick solid black lines represent the groups' pooled responses. Bars represent upper and lower limits of $95 \%$ CIs. 

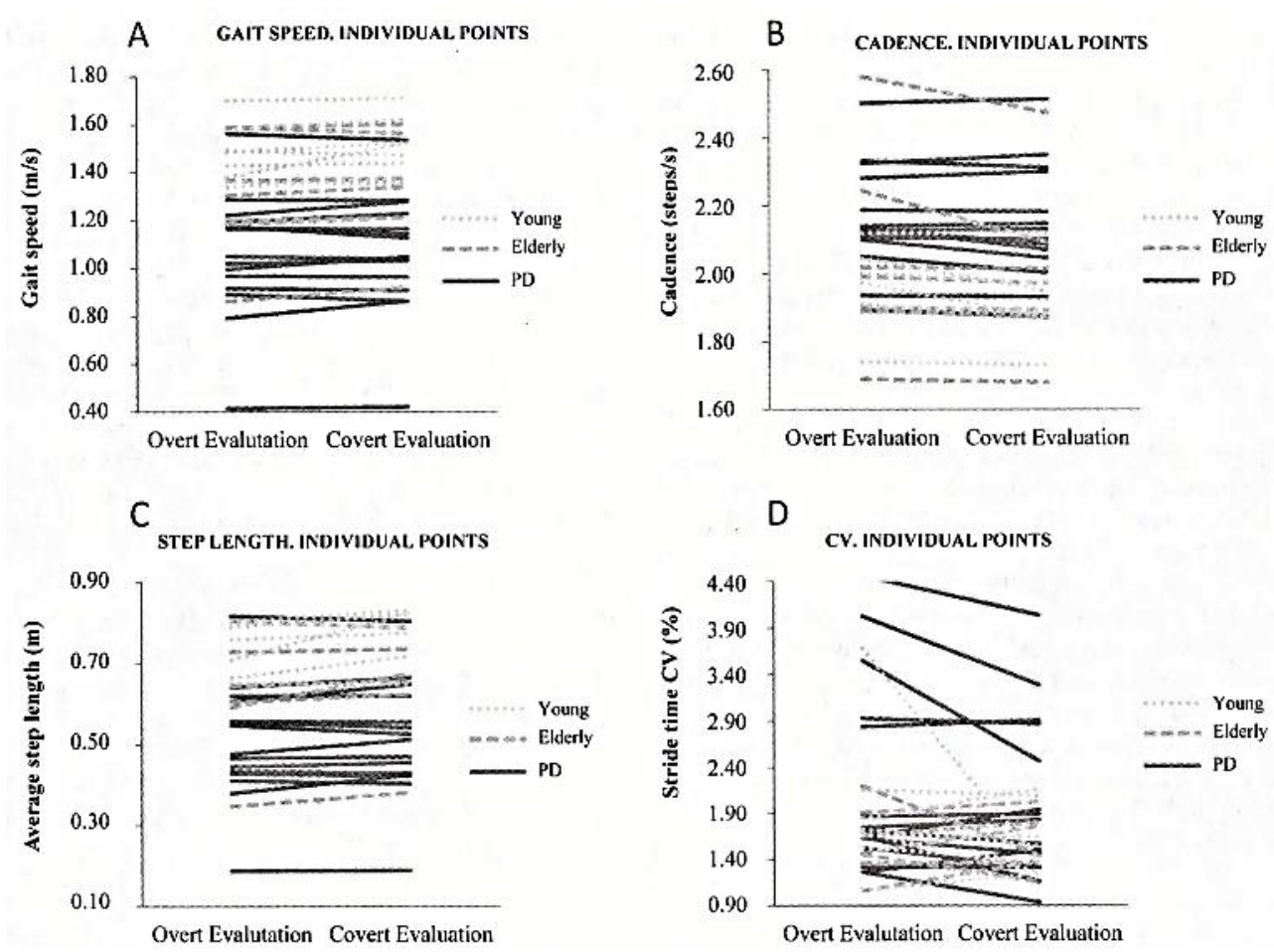

Figure 3 - Individual effect of overt and covert evaluation on gait speed (A), step cadence (B), step length (C), and $\mathrm{CV}_{\text {stride-time }}(\mathrm{D})$. The figure shows the individual PD responses (solid lines), and also individual responses for the elderly (dashed lines) and young (dotted lines) healthy subjects.

Specifically, gait speed decreased when subjects were aware of being evaluated $(1.276 \mathrm{~m} / \mathrm{s}, 95 \% \mathrm{CI}=$ $1.185,1.367)$ compared to covert evaluation $(1.294 \mathrm{~m} / \mathrm{s}, 95 \% \mathrm{CI}=1.206,1.381)$. On the other hand, when subjects were aware of evaluation, step cadence was significantly increased $(2.051 \mathrm{steps} / \mathrm{s}, 95 \% \mathrm{CI}=$ $1.978,2.124)$ compared with covert evaluation $(2.019 \mathrm{steps} / \mathrm{s}, 95 \% \mathrm{CI}=1.949,2.089)$. For average step length the pattern reversed, as this variable was significantly shorter when subjects knew the evaluation was being performed $(0.638 \mathrm{~m}, 95 \% \mathrm{CI}=0.580,0.696)$ compared to covert evaluation $(0.657 \mathrm{~m}, 95 \% \mathrm{CI}$ $=0.601,0.713)$.

Remarkably, the aforementioned effects were not significantly differentially expressed in the 3 groups included in the study, such that significant interactions (overt-covert $\mathrm{x}$ group) were not observed in gait speed $\left(\mathrm{F}_{2,27}=1.315 ; P=.285 ; \eta_{p}^{2}=.089\right)$, step cadence $\left(\mathrm{F}_{2,27}=1.615 ; P=.218 ; \eta^{2}{ }_{p}=.107\right)$, step length $\left(\mathrm{F}_{2,27}=1.881 ; P=.172 ; \eta^{2}=.122\right)$, and $\mathrm{CV}_{\text {stride-time }}\left(\mathrm{F}_{2,27}=0.610 ; P=.551 ; \eta^{2}{ }_{p}=.043\right)$.

Although the interactions of overt-covert $\mathrm{x}$ group were not statistically significant, this does not necessarily mean that the behavior of each group (how variables changed from overt to covert) has to be considered equivalent; the sample of the study seems to not be large enough to rule out the differences among groups. For instance, the largest change in gait speed covert-overt differences is present between the PD group $(0.005,95 \% \mathrm{CI}=-0.015,0.024)$ and the young controls $(0.031,95 \% \mathrm{CI}=-0.011,0.073)$, while the smallest difference between the covert-overt difference in one group and the next group for which the comparison had $95 \%$ power is 0.034 . Sample size might influence the significance of the interaction effects, such that comparisons between groups could be underpowered in all variables. Table 1 shows covert-overt differences between groups; the smallest difference needed to have $95 \%$ power for all variables. 
Table 1 Covert-overt differences for each group

\begin{tabular}{|c|c|c|c|c|c|}
\hline & Groups & $\mathrm{N}$ & Mean & $95 \% \mathrm{CI}$ & Minimum Mean Difference \\
\hline \multirow[t]{2}{*}{ Speed $(\mathrm{m} / \mathrm{s})$} & $\mathrm{PD}$ & 15 & 0.005 & $(-0.015,0.024)$ & \\
\hline & Elderly control & 7 & 0.017 & $(-0.008,0.042)$ & 0.034 \\
\hline \multirow[t]{3}{*}{ Step length (m) } & PD & 15 & 0.006 & $(-0.006,0.018)$ & \\
\hline & Elderly control & 7 & 0.022 & $(-0.007,0.051)$ & 0.026 \\
\hline & Young control & 8 & 0.030 & $(-0.004,0.063)$ & \\
\hline \multirow{2}{*}{ Step cadence (steps/s) } & Elderly control & 7 & -0.045 & $(-0.099,0.008)$ & 0.036 \\
\hline & Young control & 8 & -0.036 & $(-0.070 .-0.001)$ & \\
\hline \multirow[t]{3}{*}{$\mathrm{CV}(\%)$} & PD & 15 & -0.197 & $(-0.403,0.010)$ & \\
\hline & Elderly control & 7 & -0.026 & $(-0.380,0.329)$ & 0.462 \\
\hline & Young control & 8 & -0.329 & $(-1.02 .3,0.365)$ & \\
\hline
\end{tabular}

Note. $\mathrm{CV}=$ coefficient of variation; $\mathrm{PD}=$ Parkinson's disease

Finally, despite the fact that effects of covert and overt testing were not differently expressed in the 3 groups, the groups' behaviors were statistically different for some variables. This was the case for gait speed $\left(\mathrm{F}_{2.27}=880.1 ; P<.001 ; \eta^{2}{ }_{p}=.97\right)$. PD subjects $(1.058 \mathrm{~m} / \mathrm{s}, 95 \% \mathrm{CI}=0.939,1.177)$ were slower $(P$ $=.022)$ compared with the healthy elderly controls $(1.309 \mathrm{~m} / \mathrm{s}, 95 \% \mathrm{CI}=1.135,1.483)$, and even slower $(P$ $<.001)$ than young controls $(1.488 \mathrm{~m} / \mathrm{s}, 95 \% \mathrm{CI}=1.325,1.651$; elderly and young controls did not differ $(P=.135)$ (Figure 2A).

Likewise, step cadence varied significantly among the 3 groups $\left(\mathrm{F}_{2,27}=4.131 ; P=.027, \eta^{2}{ }_{p}=.234\right)$, but when compared in pairs, a significant difference $(P=.009)$ was only found between PD subjects $(2.151 \mathrm{steps} / \mathrm{s}, 95 \% \mathrm{CI}=2.056,2.246)$ and young controls $(1.931 \mathrm{steps} / \mathrm{s}, 95 \% \mathrm{CI}=1.800,2.061)$, and none compared with the elderly (2.023 steps/s, $95 \% \mathrm{Cl}=1.884,2.163)$ (Figure 2B).

The same group differentiations were observed for average step length $\left(\mathrm{F}_{2,27}=10.425 ; P<.001 ; \eta^{2} p\right.$ $=.436)$. Step length was shorter $(P=.016)$ in the PD group $(0.499 \mathrm{~m}, 95 \% \mathrm{CI}=0.423,0.575)$ than in the elderly $(0.669 \mathrm{~m}, 95 \% \mathrm{CI}=0.558,0.780)$, and also shorter $(P<.001)$ compared with young controls $(0.776 \mathrm{~m}, 95 \% \mathrm{CI}=0.672,0.880)$. Elderly and young controls did not differ $(P=.160)$ (Figure $2 \mathrm{C})$. 2D).

Finally, the $\mathrm{CV}_{\text {stride-time }}$ did not differ among groups $\left(\mathrm{F}_{2,27}=1.560 ; P=.229 ;=\eta^{2}{ }_{p}=.104\right)$ (Figure

\section{Discussion}

We have measured a variety of gait parameters in patients with PD and elderly and young control individuals while they were aware that measurements were being taken and compared them to measures taken covertly. Our data show that some aspects of gait kinematics are significantly influenced by overt evaluation inpatients with PD, but also in healthy subjects. ${ }^{10}$ Interestingly, the gait speed decreased subtly under overt observation, while the average step length decreased and step cadence increased. In other words, participants took smaller, faster steps when they knew they were being observed, without a large change in overall speed. $\mathrm{T} \mathrm{h}$ is suggests an interesting possibility that the subjects somehow optimized their speed, but changed strategy.

Regardless of the mean in $\mathrm{g}$ o $\mathrm{f}$ the directions of such effects, we understand that a broader message can be extracted from our results. Awareness of being evaluated modifies the subjects' gait patterns significantly, even in absence of treatment expectancy. This might condition interpretations of experimental results focused on gait impairments in different populations. 
Morris et $\mathrm{al}^{10}$ had previously evaluated overt and covert gait as part o $\mathrm{f}$ a control experiment on the therapeutic effect of visual cu e in $g$ in patients with PD. Remarkably, overt and co vert gait pattern $s$ were different in patients with PD during treatment, but absent in $\mathrm{u} n$ treated healthy controls. Therefore, a different expression of covert and overt testing in the different levels of treatment cannot be discern e d in the PD group, as a similar behavior under the same conditions in PD and healthy groups was assumed , but not evaluated.

Certain regulatory elements of gait may be automatic. ${ }^{11,12}$ Increasing complexity of second tasks while walking alters speed, step/stride cadence, and amplitude, both in PD and healthy subjects. It also seems the $\mathrm{m}$ ore complex the secondary task, the more impacted the variables. ${ }^{11}$ However, $\mathrm{CV}$ o f stride time is not affected by secondary tasks in healthy subjects, but it is in PD subjects. ${ }^{11} \mathrm{CV}$ may be considered to be a marker of gait automaticity since it changes during secondary tasks only in PD subjects, and impaired execution of automatic movements is a milestone of PD.${ }^{13}$ On this framework, it seems that the effect observed in our study focuses on the less automatic regulatory elements of gait.

On the other hand, both overt and covert evaluations differentiate groups accord in $g$ to the literature. ${ }^{3-}$ ${ }^{10}$ Notably, in our study, the variability of stride time was not different between patients with PD and healthy subjects; this is probably due to the mild severity of signs in the PD group. ${ }^{14}$

Our results suggest that covert evaluation might be useful to complement gait evaluation protocols; however, the sample size of our study was small, and for this reason this point must be confirmed in studies with larger samples. Additionally, overt and covert testing might be of interest for the study of the cognitive basis of locomotion. ${ }^{15-17}$

Our findings may have implications for the evaluation of gait procedures, which might provide a more comprehensive evaluation of the outcomes if complementing overt with covert testing. Subjects scored significantly different on several gait parameters under these testing conditions; whether the effect comes from awareness of being observed, or from being engaged in a task (regardless of being observed or not) remains to be elucidated. It seem s relevant to consider that several studies have demonstrated the critical role of a placebo on treatment effect, ${ }^{18-19}$ and also that a Hawthorne effect may reflect a situation where expectation (to perform well, to be evaluated) is also present.

This study has some limitations. The sample size used in the work is not large, particularly in regard to detecting different behavior during overt and covert observations in the different groups; however it seems that the observed effects relate to a phenomenon which might be taken into consideration during designing gait evaluation protocols. Future experiments with larger samples should try to unravel the neurobiological mechanism s implicated.

In conclusion, it could be relevant to include some covert trials during gait testing to complement the overt evaluation of so-called "spontaneous" gait.

\section{Acknowledgments}

We are indebted to Dr. Kenneth L. Grieve for the useful comments on this manuscript. This work was supported by Xunta de Galicia (Consellería de Educacion-2007/000140-0, Dirección Xeral de I+D+i; 2010-2012 and Ayudas a Grupos Consolidados, Consellería de Educación, 2013), Spain; and V. RoblesGarcía and Y. Corral-Bergantiños are funded by the FPU-MECD AP2010-2774 \& AP2010-2775, Spain. 


\section{References}

1. Schaafsma JD, Giladi N, Balash Y, Bartels AL, Gurevich T, Hausdorff JM. Gait dynamics in Parkinson's disease: relationship to Parkinsonian features, falls and response to levodopa. $J$ Neurol Sci. 2003;212(1-2):47-53. PubMed doi: 10.1016/S0022-510X(03 )00104-7

2. Seidler RD, Bernard JA. Burutolu TB, et al. Motor control and aging: links to age-related brain structural, functional, and biochemical effects. Neurosci Biobeliav Rev.2010;34(5):721—733. PubMed doi: 10.1016/j.neubiorev.2009.10.005

3. Hausdorff JM. Gait dynamics, fractals and falls: finding meaning in the stride-to-stride fluctuations of human walking. Hum Mov Sci. 2007;26(4):555-589. PubMed doi:10.1016/j.humov.2007.05.003

4. Plotnik M, Giladi N. Hausdorff JM. A new measure for quantifying the bilateral coordination of human gait: effects of aging and Parkinson's disease. Exp Brain Res. 2007; 181 (4):561-570. PubMed doi: 10.1007/S00221-007-0955-7

5. Plotnik M, Hausdorff JM. The role of gait rhythmicity and bilateral coordination of stepping in the pathophysiology of freezing of gait in Parkinson's disease. Mov Disord. 2008;23(Suppl 2):S444-S450. PubMed doi: $10.1002 / \mathrm{mds} .21984$

6. Plotnik M, Giladi N. Hausdorff JM. Bilateral coordination of walking and freezing of gait in Parkinson's disease. EurJ Neurosci. 2008;27(8): 1999-2006. PubMed doi: 10.1111/j. 14609568.2008.06167.x

7. Graham JE, Ostir GV, KuoYF. Fisher SR, Ottenbacher KJ. Relationship between test methodology and mean velocity in timed walk tests: a review. Arch Phys Med Rehabil. 2008;89(5):865-872. PubMed doi: 10.1016/j.apmr.2007.1 1.029

8. McCarney R, Warner J. Iliffe S, van Haselen R, Griffin M, Fisher P. The Hawthorne Effect: a randomised, controlled trial. BMC Med Res Methodol. 2007;7:30. PubMed doi: 10.1186/14712288-7-30

9. Parsons HM. What happened at Hawthorne?: New evidence suggests the Hawthorne effect resulted from operant reinforcement contingencies. Science. 1974; 183(4128):922—932. PubMed doi: 10.1126/science. 183.4128 .922

10. Morris ME, lansek R, Matyas TA, Summers JJ. Stride length regulation in Parkinson's disease. Brain. 1996; 119(Pt 2):551-568. PubMed doi: 10.1093/brain/119.2.551

11. Yogev G, Giladi N, Peretz C, Springer S, Simon ES, Hausdorff JM. Dual tasking, gait rhythmicity, and Parkinson's disease: which aspects of gait are attention demanding? Eur J Neurosci. 2005;22(5): 1248-1256. PubMed doi: 10.1111/j. 1460-9568.2005.04298.x

12. Dayan E, Cohen LG. N europlasticity subserving motor skill learning. Neuron. 2011;72(3):443454. PubMed doi: 10.1016/j.neuron.2011.10.008

13. Wu T, Chan P, Hallett M. Effective connectivity of neural networks in automatic movements in Parkinson's disease. Neuroimage. 2010;49(3):2581-2587. PubMed doi: 10.1016/j.neuroimage.2009.10.051

14. Arias P, Cudeiro J. Effects of rhythmic sensory stimulation (auditory, visual) on gait in Parkinson's disease patients. Exp Brain Res. 2008; 186(4):589 - 601. PubMed doi: 10.1007/s00221 -007-1263-y

15. Galati G, Lobel E, Vallar G, Berthoz A, Pizzamiglio L, Le Bihan D. The neural basis of egocentric and allocentric coding of space in humans; a functional magnetic resonance study. Exp Brain Res. 2000; 133(2): 156-164. PubMed doi: 10.1007/s002210000375

16. Kizony R, Levin MF, Hughey L, Perez C. Fung J. Cognitive load and dual-task performance during locomotion poststroke: a feasibility study using a functional virtual environment. Phys Ther. 2010;90(2):252-260. PubMed doi: 10.2522/ptj.20090061

17. Wiener JM, Berthoz A, WolbersT. Dissociable cognitive mechanisms underlying human path integration. Exp Brain Res. 2011 ;208( 1 ):61 - 71. PubMed doi: 10.1007/s00221 -0 10-2460-7

18. de la Fuente-Fernandez R, Schulzer M, Stoessl AJ, de la Fuente-Fernandez R. Placebo mechanisms and reward circuitry: clues from Parkinson's disease. Biol Psychiatry. 2004:56(2):67-71. PubMed doi: 10.1016/j. biopsych. 2003.11.019

19. Lidstone SC, Schulzer M, Dinelle K, et al. Effects of expectation on placebo-induced dopamine release in Parkinson disease. Arch Gen Psychiatry. 2010;67(8):857-865. PubMed doi: 10.1001/archgenpsy-chiatry.2010.88 